\title{
Segmentation of Color Images Using Genetic Algorithms: A Survey
}

\author{
Prerna Pachunde ${ }^{1}$, Prof.Vikal.R.Ingle ${ }^{2}$, Prof. Dr Mahindra. A. Gailwad ${ }^{3}$ \\ 1,2,3 (Department. of Electronics Engg., BDCOE,Wardha,India)
}

\begin{abstract}
Segmentation subdivides an image into its constituent regions or objects. The level to which the subdivision is carried depends on the problem being solved. Color images can increase the quality of segmentation, but increase the complexity of problem. Genetic algorithms are well suited to optimizing complex problems such as image segmentation. This paper gives state-of -art of Genetic Algorithm based segmentation methods. We discuss the feasibility of using genetic algorithms to segment general color images and discuss the issues involved in designing such algorithms.
\end{abstract}

Keywords - Color image, Genetic algorithms, Segmentation

\section{Introduction}

Image segmentation is the foundation of object recognition and computer vision. segmentation is an important process and its results are used in many image processing applications. However, there is no general way to successfully segment all images. Differing highly from grey scale images, the color segmentation usually has more complicated and time consuming algorithms and is controlled by larger set of parameters. A way of handling the complexity in color image segmentation is to use a directed search method, such as genetic algorithms. Genetic algorithms, which mimic the process of evolution, have many qualities that make them well suited to the problem of image segmentation, such as the ability to forego a local optimum to reach a global optimum[6] and the ability to efficiently find an optimal solution from within a large search space.

The main uses of genetic algorithms in image segmentation are for the modification of parameters in existing segmentation algorithms and pixel-level segmentation [15]. Various algorithms that success- fully apply genetic algorithms to image segmentation have been developed. Genetic algorithm (GA) is able to overcome many of the defects in other optimization techniques such as exhaustive techniques, calculus-based techniques, partial knowledge (hill climbing, beam search,

Due to the generality of the genetic process, they are independent of the segmentation technique used requiring only a measure of performance, which is referred to segmentation quality, for any given parameter combination. We start by looking at the concept and importance of image segmentation and mention the requirements for good image segmentation. The implication of using color in image segmentation is explored and methods for image segmentation are briefly discussed. Problems with existing image segmentation methods are mentioned. Genetic algorithms are then introduced and their suitability for use in image segmentation is examined. We explore various applications of genetic algorithms to the problem of image segmentation. Finally, the feasibility of the use of genetic algorithms for general color image segmentation is considered and design issues for such an algorithm are discussed.

\section{Equirements For ImageSegmentation}

Good image segmentationmeets certain requirements

1. Every pixel in the image belongs to a region

2. A region is connected: any two pixels in a particular region can be connected by a line that doesn't leave the region

3. Each region is homogeneous with respect to a chosen characteristic. The characteristic could be syntactic (for example, colour, intensity or texture) or based on semantic interpretation

4. Adjacent regions can't be merged into a single homogeneous region

5. No regions overlap

\section{Methods Of Image Segmentation}

Image segmentation is an old and important problem, and there are numerous image segmentation methods. Most of these methods were developed to be used on a certain class of images and there- fore aren't general image segmentation methods [6]

Bhanu and Lee [7] divide the image segmentation algorithms into three major categories: 
1. Edge Based

2. Region Based

3. Clustering Based

\subsection{Edge-Based Segmentation}

Understandably, an edge is a set of linked pixels lying on the boundary between different regions, where there are intense discontinuities such as gray change, color distinctness, texture variety and so on [6]. An image can be segmented by detecting those discontinuities. Based on this theory, there are two main edge-based segmentations methods: gray-histogram method and Gradient--based method.

The key to a satisfactory segmentation result lies in keeping a balance between detecting accuracy and noise immunity [7]. If the level of detecting accuracy is too high, noise may bring in fake edges making the outline of images unreasonable; otherwise, some parts of image outline may get undetected.

\subsection{Region-Based Segmentation}

Edge-based segmentation partitions an image based on abrupt changes in intensity near the edges whereas region- based segmentation partitions an image into regions that are similar according to a set of predefined criteria. Thresholding, region growing, region splitting and merging are the main examples of techniques in this category [10].

Region growing joins neighboring pixels with similar characteristics to form larger regions. This continues until the termination conditions are met. Most of the region growing algorithms focus on local information, making it difficult to get good global results. This method tends to excessively merge regions, resulting in under segmentation [7]. Region splitting and merging tries to over- come the weaknesses of region growing and region splitting by combining the two techniques. Initially the image is divided into arbitrary regions. Region splitting and region merging occur until the termination conditions are met [7].

\subsection{Clustering Based Techniques}

Clustering separates the image into various classes without any prior knowledge. This method is based on the assumption that objects within each class should have a high degree of similarity, while those in different classes should be dissimilar. It is considered an unsupervised image segmentation technique [12][14].

\subsection{Genetic Algorithms}

Genetic algorithms are an optimization technique used in image segmentation. It mimics natural se- lection, allowing an algorithm to adapt. Solutions are represented by a population of individual chromosomes, usually represented as binary strings. A chromosome is made up of genes, each of which can represent a particular characteristic. Each individual in the population is evaluated and given a fitness score based on how well they solve the particular problem. The higher the individual's fitness score, the greater their probability of breeding. Breeding creates the next generation through crossover and mutation. Crossover combines the chromosome of two individuals, creating a new individual which is unlike either of the parents. Mutation, which occurs only a small percent of the time, randomly alters a new individual's chromo- some. Since the more optimal individuals have a greater chance of breeding, the population tends to evolve and reach an optimal solution.

Farmer and Shugars[15] divide the genetic algorithms used for image segmentation into two major classes:

1. Parameter selection, where genetic algorithms are used to modify the parameters of an existing image segmentation method to improve its output.

2 Pixel-level segmentation, where genetic algorithms are used to perform region labeling.

Most image segmentation methods have many parameters that need to be optimized, and therefore the first method is used more often[8]. Many such methods are discussed, as well as a few methods employing pixel-level segmentation. Modified genetic algorithms and hybrid genetic algorithms have also been used for segmentation.

\section{Overview Of Ga BasedClustering Algorithms}

Cluster analysis is a technique, which is used to discover patterns and associations within data. More specifically, it is a multivariate statistical procedure that starts with a data set containing information on some variables and attempts to reorganize these data cases into relatively homogeneous groups. One of the major problems encountered by researchers, with regard to cluster analysis that different clustering methods can and do generate different solutions for the same data set. What is needed is a technique that has discovered the most 'natural' groups in a data set.

The research effort by Krovi $\mathrm{R}$. was to investigate the potential feasibility of using genetic algorithms for the purpose of clustering [5].

\subsection{Parameter Modification}


Most image segmentation methods have many parameters, constants and thresholds that need to be adjusted to produce optimal segmentation results. This creates a very large search space. Since the parameters typically interact in complex and non- linear ways, an analytic solution is not generally possible. With a reasonable amount of computation, genetic algorithms are able to find good approximations of a global optimum within a large search space. They are therefore well suited to problems involving parameter optimization. Most of the applications of genetic algorithms to image segmentation involve the optimization of various parameters[8]

Bhanu et al[8] pose image segmentation as an optimization problem. They define a general seg mentation method, whereby genetic algorithms are applied to the parameters of various well known image segmentation methods. They advocate the use of genetic algorithms to adapting the parameters of knows segmentation methods in order to be applicable to general images. They used outdoor color imagery and adapted 4 parameters of the Phoenix segmentation algorithm with genetic algorithms. They had successful results, producing high quality image segmentation with a reasonable amount of computation. Even though they perform well on outdoor scenes, these algorithms have not been proved to be able to cope with general images. The fact that these algorithms can be modified to adapt the parameters of other segmentation methods makes this method very promising.

Zingaretti [9] propose using genetic algorithms in unsupervised color image segmentation. This is another case of parameters of an existing image segmentation method being tuned by genetic algorithms. A key difference in this method is that it performs multi-pass thresholding. Different thresholds are adapted during each pass of genetic algorithms. An important advantage of this method over the previous one is that segmentation is performed totally unsupervised, without any manual segmentation. It also doesn't rely on any prior information regarding the type of image that is being processed or the task for which the segmentation results will be used. This approach successfully segmented a wide variety of images, with the exception of images that were highly textured. Pignalberi et al[8] use genetic algorithms for the optimization of parameters in an image segmentation algorithm. In this case, they focused on range images, where a pixel is colored depending on the distance between the object and a sensor. This method segments out surfaces of 3D objects, but could be applied to segmentation of 2D images.

\subsection{Pixel-Level Segmentation}

In pixel-level segmentation, genetic algorithms are used for region labeling. A pixel is classed in a particular region depending on its characteristics [15]. Peng el al[11] take this approach. Each pixel in the image is represented by a chromosome, which is a region label. The major disadvantage of this method is that the number of clusters must be given as an input, and so can't be an unsupervised, general approach. Chun and Yang[11] take a similar approach, but use a fuzzy fitness function.

\subsection{Modified Genetic Algorithms}

Gong and Yang[9] represent the image and the segmentation results by quad trees. In a similar way to Zingaretti el al they define a two pass sys- tem, genetic algorithms being used for optimization in both passes. In the first pass, genetic algorithms are used to minimize an energy function. In the optional second pass, a parameter defining how coarse or fine the segmentation is modified by genetic algorithms to obtain optimal segmentation results. The chromosomes encode the quad trees, making it inefficient to apply the usual crossover and mutation operations. To cope with this, a new crossover method and three mutation methods are defined.

Aoyagi and Tsuj use modified genetic algorithms for pixel-level segmentation. They approach image segmentation as a feature clustering problem and like Gong and Yang[9] use an energy function as a fitness function. They found it difficult to get ideal segmentation using traditional genetic algorithms, and so introduced four special types of mutation. They also propose a new method for creating individuals of the population. The segmentation problems were formulated upon such images as an optimization problem and adopt evolutionarystrategy of Genetic Algorithms for the clustering of small regions in colors feature space. The present approach uses k-Means unsupervised clustering methods into Genetic Algorithms, namely for guiding this last Evolutionary Algorithm in his search for finding the optimal or sub-optimal data partition, task that as we know, requires a non-trivial search because of its intrinsic NP-complete nature. To solve this task, the appropriate genetic coding was also discussed [13].

\subsection{Hybrid Genetic Algorithms}

Grenfenstette[20] mentions that genetic algorithms can be combined with local search techniques, creating a high performance search algorithm. The following are examples of successful implementation of hybrid genetic algorithms for image segmentation. Genetic algorithms have been combined with other evolutionary methods to tackle the image segmentation problem. Bhandarkar and Zhang[6] combine genetic algorithms with simulated annealing based techniques which mimic the physical process of cooling - to approach the problem of grey-scale image 
segmentation. They do this is an attempt to get rid of the weaknesses of each class. This resulted in better performance than traditional genetic algorithms.

\section{Applications Of Ga Based Clustering}

In previous s e cti on, various GA bas ed clustering algorithms are studied. This section provides discussion on few of applications of GA based clustering algorithms. Paper [14] shows application of a Genetic Algorithm to production simulation. The simulation is treated as a detailed, stochastic, multimodal function that describes a performance statistic. Authors tried to optimize (or at least improve) the performance of the system. A model of a real-world production line for printed circuit boards that has $\mathrm{m}$ a $\mathrm{n} y$ products and must often be retooled or reconfigured was used. Since the product line is always changing, with half of the products turning over within a year, the job of configuring and fine-tuning the production line is never ending.

\section{Conclusion}

The capability of GAs was applied to evolving the proper number of clusters and providing appropriate clustering. Many GA based clustering algorithms are studied. Some are applied on small data set and some are applied on large data set. GA based clustering techniques can be used in many application areas like production simulation, image segmentation, document clustering, image compression, gene expression analysis, text clustering etc. GA was applied on Clustering algorithms like K-means and fuzzy c-means which are mostly distance based clustering algorithms. GA is yet to be applied to other clustering algorithm.

The use of genetic algorithms in image segmentation shows promising results. Genetic algorithms are a commonly used approach to optimizing the parameters of existing image segmentation algorithms The major decisions are choosing a method of segmentation to which genetic algorithms will be applied, finding a fitness function that is a good measure of the quality of image segmentation and finding a meaningful way to represent the chromosomes.

\section{Refrences}

[1] P. D. Acton, L. S. Pilowsky, H. F. Kung, and P. J. Ell. Automatic segmentation of dynamic neuroreceptor single-photon emission tomog- raphy images using fuzzy clustering. European Journal of Nuclear Medicine, 26(6):581-590, June 1999.

[2] B. Ahrens. Genetic algorithm optimization of superresolution parameters. In Proceedings of the 2005 conference on Genetic and evolution- ary computation, GECCO, pages 2083-2088, June 2005.

[3] A. Albiol, L. Torres, and E.J. Delp. An unsu- pervised color image segmentation algorithm for facedetection applications. In Proceedings of 2001 International Conference on Image Processing, volume 2, pages 681-684, October2001.

[4] M. Alfonseca. Genetic algorithms. In Proceed- ings of the international conference on APL, pages 1-6, 1991.

[5] P. Andrey. Selectionist relaxation: Genetic algorithms applied to image segmentation. In Image and Vision Computing, volume 17, pages 175-187, 1999.

\{6] S.M. Bhandarkar and H. Zhang. Image seg- mentation using evolutionary computation. In IEEE Transactions on Evolutionary Computa-tion, volume 3, pages 1-21, April 1999.

[7] B. Bhanu and S. Lee. Genetic Learning for Adaptive Image Segmentation. Springer, 1994.

[8] B. Bhanu, S. Lee, and J. Ming. Adaptive im- age segmentation using a genetic algorithm. In IEEE Transactions on Systems, Man and Cybernetics, volume 25, pages 1543-1567, De- cember 1995.

[9] B. Bhanu and J. Peng. Adaptive integrated image segmentation and object recognition. In IEEE Transactions on Systems, Man and Cy- bernetics, Part C, volume 30, pages 427-441, November 2000.

[10] H. Chen, W. Chien, and S. Wang. Contrast- based color image segmentation. IEEE Signal Processing Letters, 11(7):641644, July 2004.

[11] D.N. Chun and H.S. Yang. Robust image seg- mentation using genetic algorithm with a fuzzy measure. In Pattern Recognition 29(7), pages 1195-1211, 1996.

[12] J. M. Daida, T. F. Bersano-Begey, and J. F. Vesechy. Computer-assisted design of image classification algorithms: Dynamic and static fitness evaluations in a scaffold genetic pro-gramming environment. In Genetic Program- ming 1996: Proceedings of the First Annual Conference, July 1996.

[13] K. S. Deshmukh and G. N. Shinde. An adap- tive color image segmentation. Electronic Let- ters on Computer Vision and Image Analysis, 5(4):12-23, 2005.

[14] G. Dong and M. Xie. Color clustering and learning for image segmentation based on neural networks. In IEEE Transactions on Neural Networks, volume 16, pages 925-936, July 2005.

[15] M.E. Farmer and D. Shugars. Application of genetic algorithms for wrapper-based im- age segmentation and classification. In IEEE Congress on Evolutionary Computation, pages 1300-1307, July 2006

[16] R.Q. Feitosa, G.A.O.P. Costa, and T.B. Cazes. A genetic approach for the automatic adapta- tion of segmentation parameters. In OBIA06,2006.

[17] S. Forrest. Genetic algorithms. ACM Computing Surveys, 28(1):77-80, March 1996.

[18] P. Ghosh and M. Mitchell. Segmentation of medical images using a genetic algorithm. In GECCO '06: Proceedings o f the 8 th annual conference on Genetic and evolutionary computation, pages 1171-1178, New York, NY, USA, 2006. ACM Press.

[19] M. Gong and Y.H. Yang. Genetic-based mul- tiresolution color image segmentation. In Vision Interface 2001, pages 141148 , June 2001.

[20] J.J. Grefenstette. Genetic algorithms and ma- chine learning. In COLT, pages 3-4, 1993. 